\title{
A Case of Painless Thyroiditis in a Very Early Stage of Pregnancy
}

\author{
Shiori Sato, Kei Endo, Toru Iizaka, Ryo Saiki, Kenji Iwaku, Shotaro Sato, \\ Yasuyoshi Takahashi, Fumiko Otsuka and Matsuo Taniyama
}

\begin{abstract}
We report a case of painless thyroiditis detected during the first trimester of pregnancy. A 29-year-old Japanese woman was hospitalized because of thyrotoxicosis and she was confirmed to be pregnant. The gestational age was 4 weeks. Blood examinations revealed negative TSH receptor antibodies, however, we started potassium iodide because we were unable to rule out Graves' disease. Thyroid hormone levels were normalized in 3 weeks and remained low even after discontinuation of medication. She received replacement therapy with levothyroxine sodium hydrate till 3 months after delivery. Painless thyroiditis can be one of the differential diagnoses of thyrotoxicosis in a very early stage of pregnancy.
\end{abstract}

Key words: thyrotoxicosis, pregnancy, painless thyroiditis

(Intern Med 51: 475-477, 2012)

(DOI: 10.2169/internalmedicine.51.5742)

\section{Introduction}

Thyrotoxicosis during pregnancy can adversely influence not only the mother but also the fetus. To avoid these influences, it is essential to identify the underlying disease responsible for thyrotoxicosis during pregnancy.

Thyrotoxicosis during the first trimester of pregnancy is mostly attributable to Graves' disease or gestational transient hyperthyroidism [GTH, also called gestational transient thyrotoxicosis (GTT)]. GTH is seen more frequently in Asians than in other races. According to a report from Belgium, the prevalence of Graves' disease is about $0.2 \%$ while that of GTH is $2 \%$ to $4 \%$ (1). Yeo et al reported that Graves's disease was seen in $1.2 \%$ and GTH in $11 \%$ of Singaporean women in the first trimester of pregnancy (2). Graves' disease requires immediate treatment, while GTH can be viewed as a physiological phenomenon that subsides spontaneously without treatment in most of the cases.

Painless thyroiditis is a disease causing thyrotoxicosis during the postpartum period or in other conditions. However, according to our literature search, no cases of painless thyroiditis developing during pregnancy have been reported, and this disease is not generally considered in the differen- tial diagnosis of thyrotoxicosis during pregnancy. Because painless thyroiditis also subsides spontaneously, it is important that this disease is distinguished from Graves' disease. We present herein a case of thyrotoxicosis detected during the first trimester of pregnancy and diagnosed with painless thyroiditis.

\section{Case Report}

The patient was a 29-year-old Japanese woman. In February 2007 , she underwent hysterosalpingography for detailed examination of infertility. She became pregnant 4 months later, but her pregnancy ended with a miscarriage at the gestational age of 5 full weeks. Nine months after the miscarriage, she became aware of palpitation, excessive sweating, and hyperphagia, followed by finger tremor and nausea. Two weeks after the appearance of these symptoms, she consulted the Showa University Fujigaoka Hospital. During the first examination, she showed slight swelling of the thyroid but she had no thyroid tenderness or vascular murmur. Exophthalmos was absent. There were no signs of heart failure. She had no family history of thyroid disease. Laboratory tests revealed thyrotoxicosis: FT3 $28.55 \mathrm{pg} / \mathrm{mL}(2.0-4.5$ $\mathrm{pg} / \mathrm{mL})$, FT4 $8.49 \mathrm{ng} / \mathrm{dL}(0.7-1.8 \mathrm{ng} / \mathrm{dL})$, and thyroid stimu- 


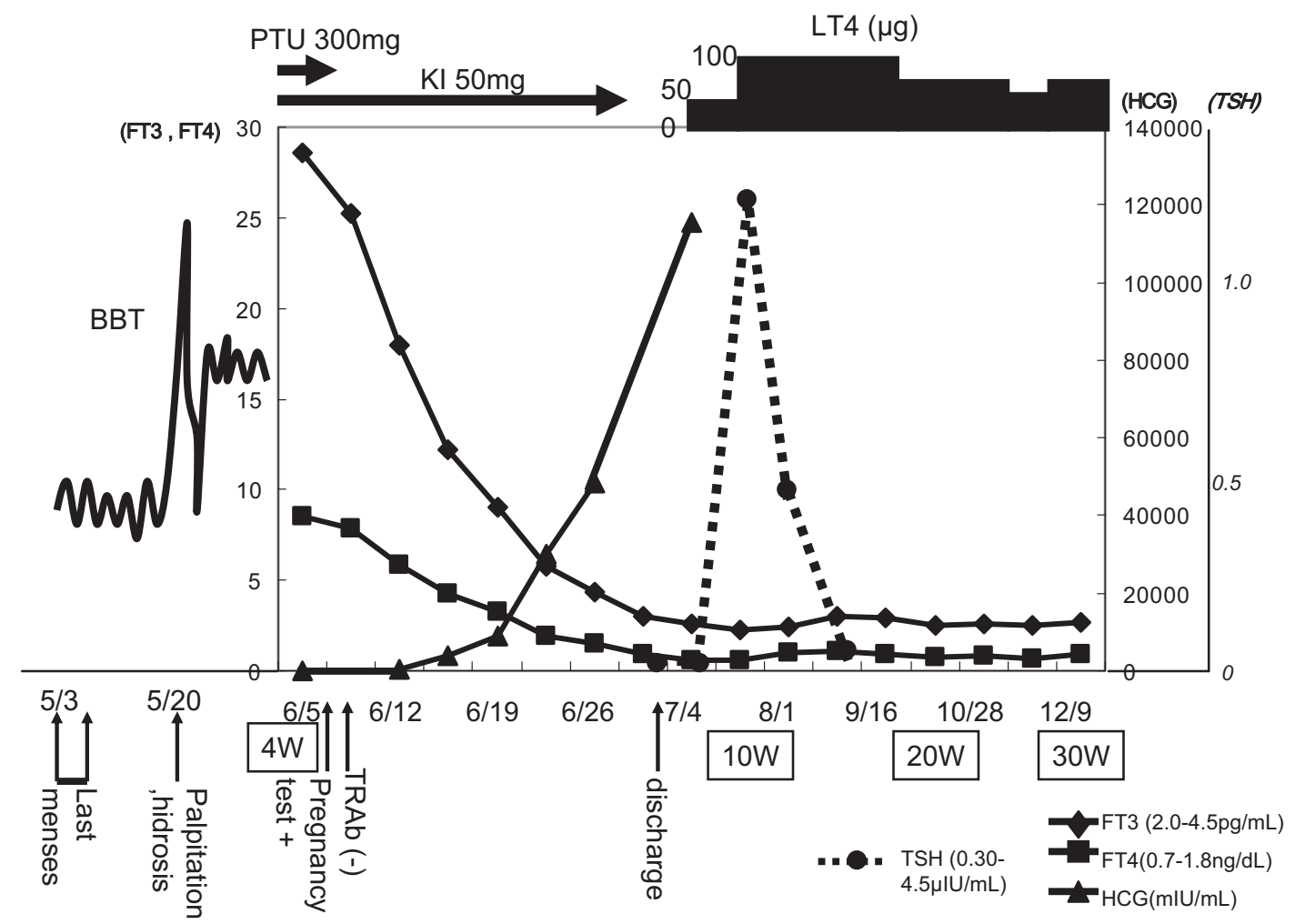

Figure 1. Clinical course of this case. $W$ : gestational age (weeks), •:TSH, $\triangle$ :HCG, n:FT4, :FT3. BBT: basal body temperature. Thyroid hormone levels normalized in approximately 1 month and remained low even after discontinuation of potassium iodide (KI) treatment.

lating hormone $(\mathrm{TSH})<0.003 \mu \mathrm{IU} / \mathrm{mL}(0.30-4.5 \mu \mathrm{IU} / \mathrm{mL})$ in early June. Other biochemical parameters suggested mild hepatic dysfunction (GOT 35 U/L [9-32], GPT 51 U/L [5-35]), accompanied by a low LDL-cholesterol level (40 mg/dL).

There was no evidence of an inflammatory reaction (white blood cells $4,200 / \mu \mathrm{L}[3,600-8,400]$, C-reactive protein $<0.2 \mathrm{mg} / \mathrm{dL}$ ). Thyroid ultrasonography revealed diffuse swelling of the thyroid gland but no signs of destructive change. Doppler study revealed no signs of increased blood flow through the thyroid.

The patient was hospitalized because of difficulty in eating due to severe nausea. Her last menstruation was in early May 2008, and we thus had to consider the possibility that she was pregnant. According to urinalysis data on admission, she was not pregnant. At that time, blood human chorionic gonadotropin (HCG) was low $(13.2 \mathrm{mIU} / \mathrm{mL})$. However, since only 4 weeks had passed since her last menstruation, pregnancy could not be ruled out. Considering the severe thyrotoxicosis and the possibility of Graves' disease, we began administering propylthiouracil (PTU, $300 \mathrm{mg} /$ day) and potassium iodide (KI, $50 \mathrm{mg} /$ day) before the results of the test for TSH receptor antibodies (TRAb) became available. On the third hospital day, her urine tested positive for pregnancy, and the patient was confirmed by the department of obstetrics to be pregnant (gestational age: about 4 weeks). The following day, TRAb was shown to be negative (TRAbhuman $<1.0 \mathrm{IU} / \mathrm{L})$. PTU was discontinued considering painless thyroiditis. However, since painless thyroiditis during pregnancy had not been previously reported, we were unable to rule out the possibility of TRAb-negative Graves' disease and thus continued KI treatment (Fig. 1). The test data obtained later revealed negative thyroid stimulating antibody $(\mathrm{TSAb})$ at $111 \%(<180)$, negative thyroid peroxidase (TPO) antibody $(<0.3 \mathrm{U} / \mathrm{mL})$, positive anti-thyroglobulin $(\mathrm{Tg})$ antibody at $15.4 \mathrm{U} / \mathrm{mL}(<0.3)$, and normal $\mathrm{Tg}$ at $16.2 \mathrm{ng} / \mathrm{mL}$ $(<30.0)$.

Three weeks later, FT3 and FT4 had normalized, and KI treatment was discontinued, allowing the patient to be discharged from the hospital. One week later, at gestational age 8 weeks, FT4 had decreased to $0.62 \mathrm{ng} / \mathrm{dL}$, which was less than the lower limit of the standard value. Although TSH had remained suppressed, the patient was interpreted to be in the hypothyroid state because TSH did not promptly respond to the reduction of thyroid hormone levels. Therefore, to avoid an adverse influence of hypothyroidism on the fetus, we began levothyroxine sodium hydrate (1-T4) administration. We initially adjusted the dose based on FT4 data and later based on TSH data (3). Her pregnancy was uneventful thereafter, and she delivered a baby weighing 2,670 $\mathrm{g}$ at gestational age 38 weeks. After delivery, the 1-T4 dose was gradually reduced, and treatment with this drug was discontinued 3 months after delivery. Thyroid hormone levels remained normal thereafter.

\section{Discussion}

Painless thyroiditis is a destructive thyroid disease with transient thyrotoxicosis usually followed by transient hypo- 
thyroidism. Postpartum painless thyroiditis is a typical form of this condition, and its onset is triggered by delivery. The onset of this disease is considered to be attributable to an immunological rebound phenomenon, i.e., sudden loss of immunological tolerance (seen during pregnancy) after delivery and the resultant activation of immune function above the normal level (4). It usually develops at 1-6 months after delivery. Cases of painless thyroiditis developing after pregnancy loss have also been reported (5).

Factors possibly triggering the onset of painless thyroiditis include a sharp reduction in high blood steroid levels [following withdrawal of steroid therapy (6), surgery for Cushing syndrome (7)] and interferon therapy (8). Changes in immune processes appear to be involved in this disease trigger, but the exact mechanism remains to be clarified.

In the present case, thyrotoxicosis was detected at a very early stage of pregnancy. Palpation and ultrasonography ruled out subacute thyroiditis or an autonomously functioning thyroid nodule. GTH was also ruled out on the basis of gestational age and the blood HCG level. As thyroid scintigraphy was not applicable in the pregnant case, we judged Graves' disease to be highly probable, because no cases of painless thyroiditis during pregnancy had been previously reported. For this reason and in view of the severe thyrotoxicosis, we began administration of PTU before the TRAb data became available. Methods for distinguishing Graves' disease from painless thyroiditis include ultrasonographic evaluation of blood flow in addition to TRAb measurement and scintigraphy. Patients with Graves' disease reportedly show increased blood flow, while those with destructive thyroiditis, such as painless thyroiditis and subacute thyroiditis, present with reduced blood flow $(9,10)$.

In the present study, TRAb was negative, and Doppler study revealed no increase in blood flow. There were few findings supporting the diagnosis of Graves' disease, and the patient showed a continuance of hypothyroidism despite medication having been discontinued after about 3 weeks of treatment for thyrotoxicosis. The patient was diagnosed as having painless thyroiditis.

Factors reportedly causing thyrotoxicosis during pregnancy include not only GTH and Graves' disease but also subacute thyroiditis and autonomously functioning thyroid nodules $(11,12)$. To date, however, no cases of painless thyroiditis during pregnancy have been reported. This may reflect the influence of immunological tolerance during pregnancy, but it is also possible that even in pregnant women with this disease, it cannot be definitively diagnosed because of inability to perform thyroid scintigraphy during pregnancy, which could account for the absence of reports on such cases.

In the present case, thyrotoxicosis was detected during pregnancy. Detection of the disease in a very early stage of pregnancy indicated, however, that the mechanism underly- ing the development of painless thyroiditis had occurred before pregnancy and that the pregnancy had been established during the course of painless thyroiditis, rather than the disease developing during pregnancy. One possible trigger for the onset of painless thyroiditis was the spontaneous abortion 9 months earlier. Marqusee et al reported cases of painless thyroiditis diagnosed from 3 to 11 months after pregnancy loss (5).

In cases with TRAb negative thyrotoxicosis during the first trimester of pregnancy, it is advisable to consider the possibility that the pregnancy was established in the presence of painless thyroiditis and to conduct detailed examinations, using Doppler ultrasonography. After these steps, it would be necessary to follow the courses of thyroid hormones closely, bearing in mind the possibility of the subsequent onset of hypothyroidism.

The authors state that they have no Conflict of Interest (COI).

\section{References}

1. Glinoer D. The regulation of thyroid function in pregnancy: pathways of endocrine adaptation from physiology to pathology. Endocr Rev 18: 404-433, 1997.

2. Yeo CP, Khoo DH, Eng PH, Tan HK, Yo SL, Jacob E. Prevalence of gestational thyrotoxicosis in Asian women evaluated in the 8th to 14th weeks of pregnancy: correlations with total and free beta human chorionic gonadotrophin. Clin Endocrinol (Oxf) 55: 391398, 2001.

3. Abalovich M, Amino N, Barbour LA, et al. Management of thyroid dysfunction during pregnancy and postpartum: an Endocrine Society Clinical Practice Guideline. J Clin Endocrinol Metab 92: S1-S47, 2007.

4. Amino N, Tada H, Hidaka Y, Izumi Y. Postpartum autoimmune thyroid syndrome. Endocr J 47: 645-655, 2000.

5. Marqusee E, Hill JA, Mandel SJ. Thyroiditis after pregnancy loss. J Clin Endocrinol Metab 82: 2455-2457, 1997.

6. Morita S, Ueda Y, Yokoyama N. Painless thyroiditis induced by the cessation of betamethasone. Intern Med 40: 744-746, 2001.

7. Takasu N, Komiya I, Nagasawa Y, Asawa T, Yamada T. Exacerbation of autoimmune thyroid dysfunction after unilateral adrenalectomy in patients with Cushing's syndrome due to an adrenocortical adenoma. N Engl J Med 322: 1708-1712, 1990.

8. Costelloe SJ, Wassef N, Schulz J, et al. Thyroid dysfunction in a UK hepatitis $\mathrm{C}$ population treated with interferon-alpha and ribavirin combination therapy. Clin Endocrinol (Oxf) 73: 249-256, 2010.

9. Ota H, Amino N, Morita S, et al. Quantitative measurement of thyroid blood flow for differentiation of painless thyroiditis from Graves' disease. Clin Endocrinol (Oxf) 67: 41-45, 2007.

10. Ito $\mathrm{Y}$, Amino N, Miyauchi A. Thyroid ultrasonography. World J Surg 34: 1171-1180, 2010.

11. Mestman JH. Hyperthyroidism in pregnancy. Best Pract Res Clin Endocrinol Metab 18: 267-288, 2004.

12. Hiraiwa $T$, Kubota $S$, Imagawa $A$, et al. Two cases of subacute thyroiditis presenting in pregnancy. J Endocrinol Invest 29: 924927, 2006.

(C) 2012 The Japanese Society of Internal Medicine http://www.naika.or.jp/imindex.html 